\begin{tabular}{|c|l|}
\hline Title & $\begin{array}{l}\text { Instability in the spectral and the Fredholm properties of an infinite dimensional Dirac operator on the abstract Boson- } \\
\text { Fermion Fock space }\end{array}$ \\
\hline Author(s) & A rai, A. \\
\hline Citation & Hokkaido University Preprint Series in Mathematics, 507, 1-6 \\
\hline Issue Date & 2000-12-1 \\
\hline DOI & 10.14943/83653 \\
\hline Doc URL & http://hdl.handle.net/2115/69257 \\
\hline Type & bulletin (article) \\
\hline File Information & pre507.pdf \\
\hline
\end{tabular}

Instructions for use 
Instability in the Spectral and the Fredholm Properties of an Infinite Dimensional Dirac Operator on the Abstract Boson-Fermion Fock Space

Asao Arai

Series \#507. December 2000 


\section{HOKKAIDO UNIVERSITY PREPRINT SERIES IN MATHEMATICS}

\#482 N. H. Bingham and A. Inoue, Tauberian and Mercerian theorems for systems of kernels, 16 pages. 2000.

\#483 N. H. Bingham and A. Inoue, Abelian, Tauberian and Mercerian theorems for arithmetic sums, 29 pages. 2000.

\#484 I. A. Bogaevski and G. Ishikawa, Lagrange mappings of the first open Whitney umbrella, 22 pages. 2000.

\#485 A. Arai and H. Kawano, A class of deformations of the Schrödinger representation of the Heisenberg commutation relation and exact solution to a Heisenberg equation and a Schrödinger equation, 22 pages. 2000 .

\#486 T. Nakazi, Functions in $N_{+}$with the positive real parts on the boundary, 21 pages. 2000.

\#487 Y. Shibukawa, Classification of the $R$-operator, 36 pages. 2000.

\#488 A. Inoue, Asymptotic behaviour for partial autocorrelation functions of fractional ARIMA processes, 20 pages. 2000.

\#489 S. Ohtani, Construction of unramified Galois extensions over maximal abelian extensions of algebraic number fields, 14 pages. 2000 .

\#490 T. Nakazi and T. Yamamoto, The real part of an outer function and a Helson-Szegö weight, 13 pages. 2000.

\#491 A. Yamagami, On Gouvêás conjecture on controlling the conductor, 11 pages. 2000.

\#492 I. Tsuda and M. Hatakeyama, Making sense of internal logic: Theory and a case study, 10 pages. 2000.

\#493 I. Tsuda, Towards an interpretation of dynamic neural activity in terms of chaotic dynamical systems, 73 pages. 2000 .

\#494 T. Mikami, Optimal control for absolutely continuous stochastic processes and the mass transportation problem, 17 pages. 2000.

\#495 M. Arisawa and Y. Giga, Anisotropic curvature flow in a very thin domain, 21 pages. 2000.

\#496 T. Nakazi, Backward shift invariant subspaces in the bidisc, 9 pages. 2000.

\#497 Y. Giga, K. Inui, J. Kato and S. Matsui, Remarks on the uniqueness of bounded solutions of the NavierStokes equations, 4 pages. 2000.

\#498 Y. Giga, M. Paolini and P. Rybka, On the motion by singular interfacial energy, 21 pages. 2000.

\#499 J. Escher and Y. Giga, On a limiting motion and self-interactions of curves moved by the intermediate surface diffusion flow, 12 pages. 2000.

\#500 I. Tsuda and S. Kuroda, Cantor coding in the hippocampus, 20 pages. 2000.

\#501 M. Tsujii, Fat solenoidal attractors, 20 pages. 2000.

\#502 A. Arai, Ground state of the massless Nelson model without infrared cutoff in a non-Fock representation, 19 pages. 2000.

\#503 Y. Giga, S. Matsui and O. Sawada, Global existence of two-dimensional Navier-Stokes flow with nondecaying initial velocity, 19 pages. 2000 .

\#504 A. Inoue and Y. Kasahara, Partial autocorrelation functions of the fractional ARIMA processes with negative degree of differencing, 14 pages. 2000.

\#505 T. Nakazi, Interpolation problem for $\ell^{1}$ and a uniform algebra, 12 pages. 2000.

\#506 R. Kobayashi and Y. Giga, On anisotropy and curvature effects for growing crystals, 38 pages. 2000. 


\title{
Instability in the Spectral and the Fredholm Properties of an Infinite Dimensional Dirac Operator on the Abstract Boson-Fermion Fock Space
}

\author{
Asao Arai * \\ Department of Mathematics, Hokkaido University \\ Sapporo 060-0810, Japan \\ Email: arai@math.sci.hokudai.ac.jp
}

November 23, 2000

to be published in the Proceedings of the Conference PARTIAL DIFFERENTIAL EQUATIONS 2000 held in Clausthal, Germany (July 24-July 28, 2000)

\begin{abstract}
A perturbed Dirac operator $Q(\alpha)$ on the abstract Boson-Fermion Fock space is considered, where $\alpha \in \mathbb{C}$ is a perturbation (coupling) parameter and the unperturbed operator $Q(0)$ is taken to be a free infinite dimensional Dirac operator introduced by the author ( A. Arai, J. Funct. Anal. 105(1992), 342-408). The following results are reported: (i) Under some conditions, the kernel of $Q(\alpha)$ is one dimensional for all $\alpha \neq \alpha_{0}$ with some $\alpha_{0} \neq 0$ and degenerate at $\alpha=\alpha_{0}$, while, under another condition, the kernel of $Q(\alpha)$ is one dimensional for all $\alpha \in \mathbb{C}$. (ii) There are cases where, for all sufficiently large $|\alpha|$ with $\alpha<0, Q(\alpha)$ has infinitely many non-zero eigenvalues even if $Q(0)$ has no non-zero eigenvalues. This is a strong coupling effect. (iii) Fredholm property of $Q(\alpha)$ also depends on the coupling parameter $\alpha$.
\end{abstract}

Key words: Boson-Fermion Fock space, supersymmetric quantum field, infinite dimensional Dirac operator, non-regular perturbation, kernel, spectrum, Fredholm property, strong coupling effect

${ }^{*}$ Supported by the Grant-In-Aid No.11440036 for Scientific Research from the Ministry of Education, Science, Sports and Culture, Japan. 


\section{Introduction}

Let $\mathcal{H}$ and $\mathcal{K}$ be separable complex Hilbert spaces. Then the abstract Boson-Fermion Fock space $\mathcal{F}(\mathcal{H}, \mathcal{K})$ associated with the pair $\langle\mathcal{H}, \mathcal{K}\rangle$ is defined by

$$
\mathcal{F}(\mathcal{H}, \mathcal{K}):=\mathcal{F}_{\mathbf{b}}(\mathcal{H}) \otimes \mathcal{F}_{\mathrm{f}}(\mathcal{K})
$$

where $\mathcal{F}_{\mathrm{b}}(\mathcal{H}):=\oplus_{n=0}^{\infty} \otimes_{\mathrm{s}}^{n} \mathcal{H}$ is the Boson Fock space over $\mathcal{H}\left(\otimes_{\mathrm{s}}^{n} \mathcal{H}\right.$ denotes the $n$-fold symmetric tensor product Hilbert space of $\left.\mathcal{H} ; \otimes_{\mathbf{s}}^{0} \mathcal{H}:=\mathbb{C}\right)$ and $\mathcal{F}_{\mathbf{f}}(\mathcal{K}):=\oplus_{p=0}^{\infty} \wedge^{p} \mathcal{K}$ is the Fermion Fock space over $\mathcal{K}\left(\wedge^{p} \mathcal{K}\right.$ denotes the $p$-fold anti-symmetric tensor product Hilbert space of $\left.\mathcal{K} ; \wedge^{0} \mathcal{K}:=\mathbb{C}\right)$.

Let $\mathrm{C}(\mathcal{H}, \mathcal{K})$ be the set of densely defined closed linear operators from $\mathcal{H}$ to $\mathcal{K}$. Then, for each $A \in \mathrm{C}(\mathcal{H}, \mathcal{K})$, one can define a Dirac-type operator $Q_{A}$ on $\mathcal{F}(\mathcal{H}, \mathcal{K})$ [Ar92] (for the definition of $Q_{A}$, Section 2 below). The operator $Q_{A}$ is an infinite dimensional version of free Dirac operators on finite dimensional spaces.

In [Ar92] some fundamental properties of $Q_{A}$ were established. Moreover, a perturbed Dirac operator of the form $Q_{A}(V):=Q_{A}+V$ was considered in view of index theory, where $V$ is a symmetric operator on $\mathcal{F}(\mathcal{H}, \mathcal{K})$, and a functional integral representation for the index of $Q_{A}(V)$ restricted to a subspace, called the "bosonic subspace", was derived (for related aspects and further developments, see [Ar89], [Ar91], [Ar93], [Ar93], [Ar94], [Ar96], [Ar97], [AM91], [AM93]). It still remains, however, as an important problem, to investigate spectral properties of $Q_{A}(V)$. In this paper, as a first step towards this direction, we present a perturbation of $Q_{A}$ which is not of the form $V$ considered in [Ar92] and rather simple, but, gives rise to interesting nonperturbative instability phenomena in spectral and Fredholm properties. For proofs on the results reported in this paper, see [Ar00].

\section{A Class of Perturbed Dirac Operators}

We denote by $a(f)(f \in \mathcal{H})$ and $b(u)(u \in \mathcal{K})$ the annihilation operators on $\mathcal{F}_{\mathrm{b}}(\mathcal{H})$ and on $\mathcal{F}_{\mathrm{f}}(\mathcal{K})$ respectively (e.g., $[\mathrm{BR} 97, \S 5.2]$ ). Let $\Omega_{\mathrm{b}}:=\{1,0,0, \cdots\} \in \mathcal{F}_{\mathrm{b}}(\mathcal{H})$ (resp. $\Omega_{\mathrm{f}}:=$ $\left.\{1,0,0, \cdots\} \in \mathcal{F}_{\mathbf{f}}(\mathcal{K})\right)$ be the Fock vacuum in $\mathcal{F}_{\mathrm{b}}(\mathcal{H})\left(\right.$ resp. $\left.\mathcal{F}_{\mathrm{f}}(\mathcal{K})\right)$. Let $A \in \mathrm{C}(\mathcal{H}, \mathcal{K})$ and

$$
\begin{gathered}
\mathcal{D}_{A}^{\infty}:=\mathcal{L}\left\{a\left(f_{1}\right)^{*} \cdots a\left(f_{n}\right)^{*} \Omega_{\mathrm{b}} \otimes b\left(u_{1}\right)^{*} \cdots b\left(u_{p}\right)^{*} \Omega_{\mathrm{f}} \mid n, p \geq 0, f_{j} \in C^{\infty}\left(A^{*} A\right)\right. \\
\left.j=1, \cdots, n, u_{k} \in C^{\infty}\left(A A^{*}\right), k=1, \cdots, p\right\}
\end{gathered}
$$

where $\mathcal{L}\{\cdots\}$ means the subspace algebraically spanned by the vectors in the set $\{\cdots\}$ and $C^{\infty}(T):=\cap_{n=1}^{\infty} D\left(T^{n}\right)$ for a linear operator $T$ on a Hilbert space $\left(D\left(T^{n}\right)\right.$ denotes the domain of $T^{n}$ ). It follows that $\mathcal{D}_{A}^{\infty}$ is dense in $\mathcal{F}(\mathcal{H}, \mathcal{K})$.

Let $\left\{e_{n}\right\}_{n=1}^{\infty}$ be a complete orthonormal system of $\mathcal{K}$ such that $e_{n} \in D\left(A^{*}\right), n \in \mathbb{N}$. Then it is shown that there is a unique densely defined closed linear operator $d_{A}$ on $\mathcal{F}(\mathcal{H}, \mathcal{K})$ such that (i) for all $\Psi \in \mathcal{D}_{A}^{\infty}$

$$
d_{A} \Psi=\sum_{n=1}^{\infty} a\left(A^{*} e_{n}\right) b\left(e_{n}\right)^{*} \Psi
$$


independently of the choice of $\left\{e_{n}\right\}_{n=1}^{\infty}$ and (ii) $\mathcal{D}_{A}^{\infty}$ is a core of $d_{A}$. It follows that $d_{A}^{2}=0$. The operator may be regarded as an infinite dimensional version of finite dimensional exterior differential operators. A free Dirac operator on $\mathcal{F}(\mathcal{H}, \mathcal{K})$ is defined by

$$
Q_{A}=d_{A}+d_{A}^{*}
$$

We have an orthogonal decomposition

$$
\mathcal{F}(\mathcal{H}, \mathcal{K})=\mathcal{F}_{+}(\mathcal{H}, \mathcal{K}) \oplus \mathcal{F}_{-}(\mathcal{H}, \mathcal{K})
$$

with

$$
\mathcal{F}_{+}(\mathcal{H}, \mathcal{K}):=\oplus_{p=0}^{\infty} \mathcal{F}_{\mathrm{b}}(\mathcal{H}) \otimes \wedge^{2 p}(\mathcal{K}), \quad \mathcal{F}_{-}(\mathcal{H}, \mathcal{K}):=\oplus_{p=0}^{\infty} \mathcal{F}_{\mathrm{b}}(\mathcal{H}) \otimes \wedge^{2 p+1}(\mathcal{K})
$$

Let $P_{+}$and $P_{-}$be the orthogonal projections onto $\mathcal{F}_{+}(\mathcal{H}, \mathcal{K})$ and $\mathcal{F}_{-}(\mathcal{H}, \mathcal{K})$ respectively and define

$$
\Gamma:=P_{+}-P_{-} \text {. }
$$

For a self-adjoint operator $S$ on $\mathcal{H}(\operatorname{resp} . \mathcal{K}), d \Gamma_{\mathbf{b}}(S)\left(\right.$ resp. $\left.d \Gamma_{\mathbf{f}}(S)\right)$ denotes the second quantization of $S$ in the Boson Fock space $\mathcal{F}_{\mathbf{b}}(\mathcal{H})$ (resp. the Fermion Fock space $\mathcal{F}_{\mathbf{f}}(\mathcal{H})$ ) [BR97, §5.2].

Basic properties of $Q_{A}$ are as follows [Ar92]:

(i) The operator $Q_{A}$ is self-adjoint and essentially self-adjoint on $\mathcal{D}_{A}^{\infty}$.

(ii) The operator $\Gamma$ leaves $D\left(Q_{A}\right)$ invariant and $\Gamma Q_{A}+Q_{A} \Gamma=0$ on $D\left(Q_{A}\right)$.

(iii) The following operator equations hold :

$$
Q_{A}^{2}=d_{A}^{*} d_{A}+d_{A} d_{A}^{*}=d \Gamma_{\mathrm{b}}\left(A^{*} A\right) \otimes I+I \otimes d \Gamma_{\mathrm{f}}\left(A A^{*}\right),
$$

where $I$ denotes identity.

We perturb $Q_{A}$ through a perturbation of $d_{A}$. Let $g \in D(A), g \neq 0$, and $v \in$ $D\left(A^{*}\right), v \neq 0$ and define

$$
d(\alpha):=d_{A}+\alpha a(g) \otimes b(v)^{*} .
$$

where $\alpha \in \mathbb{C}$ is a coupling constant. It is easy to see that $D(d(\alpha)) \supset \mathcal{D}_{A}^{\infty}$ and $d(\alpha) \mid \mathcal{D}_{A}^{\infty}$ is closable. Let

$$
\bar{d}(\alpha):=\overline{d(\alpha) \mid \mathcal{D}_{A}^{\infty}},
$$

the closure of $d(\alpha) \mid \mathcal{D}_{A}^{\infty}$, and

$$
Q(\alpha):=\bar{d}(\alpha)+\bar{d}(\alpha)^{*}
$$

This is the perturbed Dirac operator considered in this paper. 


\section{Results}

Theorem 3.1 (i) For all $\alpha \in \mathbb{C}, Q(\alpha)$ is self-adjoint, and essentially self-adjoint on $\mathcal{D}_{A}^{\infty}$ with $Q(\alpha)=\overline{Q_{A}+V_{g, v}}$, where $V_{g, v}:=\alpha a(g) \otimes b(v)^{*}+\alpha^{*} a(g)^{*} \otimes b(v)$. Moreover, $\Gamma$ leaves $D(Q(\alpha))$ invariant and $\Gamma Q(\alpha)+Q(\alpha) \Gamma=0$ on $D(Q(\alpha))$.

(ii) Suppose that $A$ is injective and $g \in D\left(|A|^{-1}\right)$. Then, for all $|\alpha|<\left(\|v\|\left\||A|^{-1} g\right\|\right)^{-1}$, $D(Q(\alpha))=D\left(Q_{A}\right)$.

(iii) For all $z \in \mathbb{C} \backslash \mathbb{R},(Q(\alpha)-z)^{-1}$ is strongly continuous in $\alpha \in \mathbb{C}$.

To describe the spectral properties of $Q(\alpha)$, we introduce a bounded linear operator $T_{g, v}$ from $\mathcal{H}$ to $\mathcal{K}$ by

$$
T_{g, v} f:=(g, f) v, \quad f \in \mathcal{H},
$$

where $(\cdot, \cdot)$ denotes inner product, and define

$$
A(\alpha):=A+\alpha T_{g, v}
$$

For a linear operator $T$ on a Hilbert space, we denote by $\sigma(T)$ (resp. $\sigma_{\mathbf{p}}(T)$ ) the (resp. point) spectrum of $T$. A general feature of the spectra of $Q(\alpha)$ is given in the following theorem:

Theorem 3.2 For all $\alpha \in \mathbb{C}, \sigma(Q(\alpha))$ and $\sigma_{\mathrm{p}}(Q(\alpha))$ are symmetric with respect to the origin and

$$
\begin{aligned}
& \sigma(Q(\alpha))=\{0\} \bigcup\left(\bigcup_{n=1}^{\infty}\left\{ \pm \sqrt{\sum_{j=1}^{n} \lambda_{j} \mid} \lambda_{j} \in \sigma\left(A(\alpha)^{*} A(\alpha)\right), j=1, \cdots, n\right\}\right) \\
& \sigma_{\mathrm{p}}(Q(\alpha))=\{0\} \bigcup\left(\bigcup_{n=1}^{\infty}\left\{ \pm \sqrt{\sum_{j=1}^{n} \lambda_{j}} \mid \lambda_{j} \in \sigma_{\mathbf{p}}\left(A(\alpha)^{*} A(\alpha)\right), j=1, \cdots, n\right\}\right)
\end{aligned}
$$

with

$$
\operatorname{dim} \operatorname{ker}(Q(\alpha)-\lambda)=\operatorname{dim} \operatorname{ker}(Q(\alpha)+\lambda), \quad \lambda \in \sigma_{\mathbf{p}}(Q(\alpha))
$$

This theorem shows that the spectrum and the point spectrum of $Q(\alpha)$ are completely determined from those of $A(\alpha)^{*} A(\alpha)$.

To state properties of the kernel of $Q(\alpha)$, we introduce the following conditions on $\{A, g, v\}$ :

(C.1) $A$ is injective and $v \in D\left(A^{-1}\right)$ with $\left(g, A^{-1} v\right) \neq 0$. In this case we introduce a constant

$$
\alpha_{0}:=-\frac{1}{\left(g, A^{-1} v\right)} .
$$

(C.2) $A^{*}$ is injective and $g \in D\left(A^{*-1}\right)$ with $\left(A^{*-1} g, v\right) \neq 0$. In this case we introduce a constant

$$
\beta_{0}:=-\frac{1}{\left(A^{*-1} g, v\right)} .
$$


(C.3) $A$ is injective, and $v \notin D\left(A^{-1}\right)$ or $v \in D\left(A^{-1}\right)$ with $\left(g, A^{-1} v\right)=0$.

(C.4) $A^{*}$ is injective, and $g \notin D\left(A^{*-1}\right)$ or $g \in D\left(A^{*-1}\right)$ with $\left(A^{*-1} g, v\right)=0$.

For a linear operator $T$ on a Hilbert space, we $\operatorname{set} \operatorname{nul} T:=\operatorname{dim} \operatorname{ker} T$.

Theorem 3.3 (i) Suppose that (C.1) and (C.2) hold. Let

$$
\Psi_{n, j}:=a\left(A^{-1} v\right)^{*^{n}} \Omega_{\mathrm{b}} \otimes b\left(A^{*-1} g\right)^{*^{j}} \Omega_{\mathrm{f}}, \quad n=0,1,2, \cdots, \quad j=0,1 .
$$

Then nul $Q\left(\alpha_{0}\right)=\infty$ with $\operatorname{ker} Q\left(\alpha_{0}\right)=\overline{\mathcal{L}\left\{\Psi_{n, j} \mid n \geq 0, j=0,1\right\}}$. Moreover, for all $\alpha \neq \alpha_{0}$,

$$
\operatorname{nul} Q(\alpha)=1, \quad \operatorname{ker} Q(\alpha)=\left\{c \Omega_{\mathrm{b}} \otimes \Omega_{\mathrm{f}} \mid c \in \mathbb{C}\right\} .
$$

(ii) Suppose that (C.1) and (C.4) hold. Then nul $Q\left(\alpha_{0}\right)=\infty$ with

$$
\operatorname{ker} Q\left(\alpha_{0}\right)=\overline{\mathcal{L}\left\{\Psi_{n, 0} \mid n \geq 0\right\}} \text {. }
$$

Moreover, for all $\alpha \neq \alpha_{0}$, (3.9) holds.

(iii) Suppose that (C.2) and (C.3) hold. Then nul $Q\left(\beta_{0}\right)=2$ with $\operatorname{ker} Q\left(\beta_{0}\right)=\mathcal{L}\left\{\Psi_{0, j} \mid j=\right.$ $0,1\}$.

(iv) Suppose that (C.3) and (C.4) hold. Then, for all $\alpha \in \mathbb{C}$, (3.9) holds.

As for non-zero eigenvalues of $Q(\alpha)$, we have the following result:

Theorem 3.4 Consider the case where $\mathcal{H}=\mathcal{K}, A$ is a nonnegative self-adjoint operator with $\operatorname{ker} A=\{0\}$ and $g=v \in D\left(A^{-1}\right)$ (then $\left.\alpha_{0}=-1 /\left(v, A^{-1} v\right)<0\right)$. Let $\alpha<\alpha_{0}$. Then, there exists a constant $x_{0}(\alpha)<0$ such that $\alpha\left(v,\left(x_{0}(\alpha)-A\right)^{-1} v\right)=1$, and for all $n \in\{0\} \cup \mathbb{N}$,

$$
\pm \sqrt{n}\left|x_{0}(\alpha)\right| \in \sigma_{\mathrm{p}}(Q(\alpha)) .
$$

Note that this theorem holds even if $Q_{A}$ has no non-zero eigenvalues. As the condition that $\alpha<\alpha_{0}$ shows, this is a strong coupling effect.

By Theorem 3.1 (i)-(ii), the operator $Q_{+}(\alpha)$ defined by

$$
D\left(Q_{+}(\alpha)\right):=D(Q(\alpha)) \cap \mathcal{F}_{+}(\mathcal{H}, \mathcal{K}), \quad Q_{+}(\alpha) \Psi:=Q(\alpha) \Psi, \Psi \in D\left(Q_{+}(\alpha)\right)
$$

is a densely defined closed linear operator from $\mathcal{F}_{+}(\mathcal{H}, \mathcal{K})$ to $\mathcal{F}_{-}(\mathcal{H}, \mathcal{K})$. We define an index of $Q(\alpha)$ by

$$
\operatorname{ind}_{\Gamma}(Q(\alpha)):=\operatorname{nul} Q_{+}(\alpha)-\operatorname{nul} Q_{+}(\alpha)^{*},
$$

the index of $Q_{+}(\alpha)$, provided that nul $Q_{+}(\alpha)<\infty$ or nul $Q_{+}(\alpha)^{*}<\infty$.

Results on the (semi-)Fredholm property and the index ind $\operatorname{di}_{\Gamma} Q(\alpha)$ are as follows:

Theorem 3.5 (i) Suppose that (C.1) and (C.2) hold. Then $Q\left(\alpha_{0}\right)$ is not semi-Fredholm. Moreover, for all $\alpha \neq \alpha_{0}, Q(\alpha)$ is Fredholm with $\operatorname{ind}_{\Gamma} Q(\alpha)=1$. 
(ii) Suppose that (C.1) and (C.4) hold. Then $Q\left(\alpha_{0}\right)$ is semi-Fredholm with ind $\operatorname{lo}_{\Gamma}(\alpha)=$ $\infty$. Moreover, for all $\alpha \neq \alpha_{0}, Q(\alpha)$ is Fredholm with $\operatorname{ind}_{\Gamma} Q(\alpha)=1$.

(iii) Suppose that (C.2) and (C.3) hold. Then $Q\left(\beta_{0}\right)$ is Fredholm with ind $\Gamma Q\left(\beta_{0}\right)=0$. Moreover, for all $\alpha \neq \beta_{0}, Q(\alpha)$ is Fredholm with ind ${ }_{\Gamma} Q(\alpha)=1$.

(iv) Suppose that (C.3) and (C.4) hold. Then, for all $\alpha \in \mathbb{C}, Q(\alpha)$ is Fredholm with $\operatorname{ind}_{\Gamma} Q(\alpha)=1$.

\section{References}

[Ar89] A. Arai, Path integral representation of the index of Kähler-Dirac operators on an infinite dimensional manifold, J. Funct. Anal. 82(1989), 330-369.

[Ar91] A. Arai, A general class of infinite dimensional Dirac operators and related aspects, Functional Analysis \& Related Topics (Ed. S. Koshi), pages 85-98, World Scientific, Singapore, 1991.

[Ar92] A. Arai, A general class of infinite dimensional Dirac operators and path integral representation of their index, J. Funct. Anal. 105(1992), 342-408.

[Ar93] A. Arai, Dirac operators in Boson-Fermion Fock spaces and supersymmetric quantum field theory, J. Geom. Phys. 11(1993), 465-490.

[Ar93] A. Arai, Supersymmetric extension of quantum scalar field theories, Quantum and Non-Commutative Analysis (Ed. H.Araki et al), pages 73-90, Kluwer Academic Publishers, Dordrecht 1993.

[Ar94] A. Arai, On self-adjointness of Dirac operators in boson-fermion Fock spaces, Hokkaido Math. Jour. 23(1994), 319-353.

[Ar96] A. Arai, Supersymmetric quantum field theory and infinite dimensional analysis, Sugaku Expositions 9(1996), 87-98.

[Ar97] A. Arai, Strong anticommutativity of Dirac operators on Boson-Fermion Fock spaces and representations of a supersymmetry algebra, Math. Nachr. 207 (1999), $61-77$.

[Ar00] A. Arai, Spectral properties of a Dirac operator on the abstract Boson-Fermion Fock space, in preparation.

[AM91] A. Arai and I. Mitoma, De Rham-Hodge-Kodaira decompositionin $\infty$ - dimensions, Math. Ann. 291 (1991), 51-73.

[AM93] A. Arai and I. Mitoma, Comparison and nuclearity of spaces of differential forms on topological vector spaces, J. Funct. Anal. 111(1993), 278-294.

[BR97] O. Bratteli and D. W. Robinson, Operator Algebras and Quantum Statistical Mechanics 2, Second Edition, Springer, Berlin, Heidelberg, 1997. 